\title{
A Class of Linearly Implicit Numerical Methods for Solving Stiff Ordinary Differential Equations
}

\author{
S.S. Filippov* and A.V. Tygliyan
}

Keldysh Institute of Applied Mathematics, Russian Academy of Sciences, Moscow 125047, Russia

\begin{abstract}
We introduce $A B C$-schemes, a new class of linearly implicit one-step methods for numerical integration of stiff ordinary differential equation systems. Formulas of $A B C$-schemes invoke the Jacobian of differential system similary to the methods of Rosenbrock type, but unlike the latter they include also the square of the Jacobian matrix.
\end{abstract}

Keywords: Solving stiff ordinary differential equations, Linearly implicit methods, $A B C$-schemes, $A$-stability, $L$-stability.

\section{INTRODUCTION}

We propose a new class of one-step numerical methods for solving stiff ordinary differential equations. These methods employ the Jacobian of a differential system and, in distinction from Rosenbrock methods [1], the square of Jacobian is also involved in their formulas. The first two onestage methods of this kind were reported by S.S. Filippov and M.V. Bulatov (Conference on Scientific Computation, Geneva, Switzerland, June 26-29, 2002, p. 26); see also [2]. The term ' $A B C$-schemes' for such methods was suggested later in [3].

In Section 2 one-stage $A B C$-schemes are defined and some results obtained for them are presented. Section 3 contains several examples of one-stage $A B C$-schemes. Multistage $A B C$-schemes are introduced in Section 4. Two examples of two-stage $A B C$-schemes are given in Section 5. Some results of a numerical experiment with $A B C$-schemes compared with those obtained by the use of implicit RungeKutta methods are presented in Section 6.

\section{ONE-STAGE $A B C$-SCHEMES}

\section{Definition 1}

A one-stage $A B C$-scheme for numerical integration of a Cauchy problem for an autonomous system of $n$ ordinary differential equations

$y^{\prime}(x)=f(y(x)), \quad y\left(x_{0}\right)=y_{0}$

is defined as follows:

$\left(I+A h f_{y}+B h^{2} f_{y}^{2}\right)\left[y_{1}(h)-y_{0}\right]=\left(I+C h f_{y}\right) h f \quad \ldots$

Here, $A, B$, and $C$ are the coefficients that determine a particular method, $y_{1}(h)$ is the desired numerical solution after one step of integration with the step size $h, y(x)$ and

*Address correspondence to this author at the Keldysh Institute of Applied Mathematics of the Russian Academy of Sciences, Miusskaya sq. 4, Moscow 125047, Russia; Tel: +7(495)2507985; Fax: +7(499)9720737;

E-mail: filippov@ keldysh.ru $f(y)$ are $n$-dimensional vector functions, $f_{y}$ is the Jacobian matrix, and $I$ is the identity matrix. We consider the first step of integration as a representative one for the subsequent steps and write $f, f_{y}, \ldots$ without arguments for $f\left(y_{0}\right)$, $f_{y}\left(y_{0}\right), \ldots$.

The following statements for one-stage $A B C$-schemes can be easily proved in standard way (see e.g. [4] and [5]).

\section{Theorem 1}

The convergence order of methods (2) is not less then one at any choice of real coefficients $A, B$, and $C$.

\section{Theorem 2}

The order of methods (2) equals two, iff $C=A+\frac{1}{2}$.

In this case, the principal error term is equal to $y\left(x_{0}+h\right)-y_{1}(h)=\frac{h^{3}}{3 !}\left(f_{y y} f f+\sigma f_{y}^{2} f\right)$,

where

$\sigma=1+3 A+6 B$

and

$f_{y y} f f=\sum_{j, k=1}^{n} \frac{\partial^{2} f_{i}}{\partial y_{j} \partial y_{k}} f_{j} f_{k}$,

$f_{y}^{2} f=\sum_{j, k=1}^{n} \frac{\partial f_{i}}{\partial y_{j}} \frac{\partial f_{j}}{\partial y_{k}} f_{k}$

for $i=1, \ldots, n$.

\section{Theorem 3}

The stability function of $A B C$-schemes (2) is given by

$R(z)=\frac{1+(1+A) z+(B+C) z^{2}}{1+A z+B z^{2}}$.

2010 Bentham Open 


\section{Theorem 4}

The $A B C$-schemes (2) of order two are $A$-stable, iff

$A \leq-\frac{1}{2}, \quad B \geq-\frac{A}{2}-\frac{1}{4}$.

\section{Theorem 5}

The $A B C$-schemes (2) of order two are $L$-stable, iff

$B=-A-\frac{1}{2}$.

Furthermore, some important results for linear autonomous systems follow immediately from the above theorems.

\section{Corollary 1}

$A B C$-schemes (2) approximate solutions to linear systems (1) having constant coefficients with order three, iff

$B=-\frac{A}{2}-\frac{1}{6}, \quad A \leq-\frac{1}{2}$.

In this case, Eq. (3) yields $\sigma=0$, and we have a family of methods depending on the single parameter $A$ :

$\left[I+A h f_{y}-\left(\frac{A}{2}+\frac{1}{6}\right) h^{2} f_{y}^{2}\right]\left(y_{1}(h)-y_{0}\right)=h f+\left(A+\frac{1}{2}\right) h^{2} f_{y} f$

with the principal error term

$y\left(x_{0}+h\right)-y_{1}(h)=\frac{h^{4}}{4 !}(1+2 A) f_{y}^{3} f$

and stability function

$$
R(z)=\frac{1+(1+A) z+\left(\frac{A}{2}+\frac{1}{3}\right) z^{2}}{1+A z-\left(\frac{A}{2}+\frac{1}{6}\right) z^{2}} .
$$

These methods are $A$-stable for $A \leq-1 / 2$, and at $A=-2 / 3$ the method is also $L$-stable.

\section{Corollary 2}

With $A=-1 / 2$ method (4) takes the form

$$
\left(I-\frac{1}{2} h f_{y}+\frac{1}{12} h^{2} f_{y}^{2}\right)\left(y_{1}(h)-y_{0}\right)=h f .
$$

It gives fourth order approximation for the solutions of linear autonomous systems of differential equations. Its principal term of local error is then equal to

$y\left(x_{0}+h\right)-y_{1}(h)=\frac{h^{5}}{5 !} \frac{1}{6} f_{y}^{4} f$.

This method is $A$-stable with the stability function

$$
R(z)=\frac{1+\frac{1}{2} z+\frac{1}{12} z^{2}}{1-\frac{1}{2} z+\frac{1}{12} z^{2}} .
$$

\section{Remark 1}

Solving linear system of algebraic equations (2) in its original form seems to be rather expensive. Indeed, in addition to $\sim n^{3} / 3$ multiplications and divisions that are needed for the $L U$ - decomposition of the matrix in the left-hand side of Eq. (2), extra $n^{3}$ multiplications are required for squaring a full matrix $f_{y}$ of $n$th order, i.e. totally $\sim 4 n^{3} / 3$ multiplicative operations. However, it is possible to avoid matrix multiplication by decomposing the matrix in the left-hand side of Eq. (2) as follows:

$$
I+A h f_{y}+B h^{2} f_{y}^{2}=\left(I+F h f_{y}\right)\left(I+G h f_{y}\right)
$$

where the new coefficients $F$ and $G$ are real or complex numbers depending on the values of $A$ and $B$. In this case, only two $L U$ - decompositions of the matrices $\left(I+F h f_{y}\right)$ and $\left(I+G h f_{y}\right)$ are needed, i.e. totally $\sim 2 n^{3} / 3$ multiplicative operations. Moreover, the amount of arithmetical operations can be once more halved, if we confine ourselves with the choice $B=A^{2} / 4$, because in this case $F=G=A / 2$, and only one $L U$ - decomposition is needed ('cheap' $A B C$ schemes).

Fig. (1) illustrates some essential results obtained for the one-stage $A B C$-schemes of order 2. Region $\boldsymbol{A}$ contains all pairs of coefficients $(A, B)$ corresponding to $A$-stable methods (Theorem 4). The thick line $\boldsymbol{L}$ indicates $L$-stable methods (Theorem 5). The dashed line is the locus of all the methods (4) with $\sigma=0$ (Corollary 1). The dotted parabola $B=A^{2} / 4$ represents the 'cheap' $A B C$-schemes (Remark 1). The examples from the next section are indicated by small circles with corresponding numbers.

\section{EXAMPLES OF ONE-STAGE $A B C$-SCHEMES}

Each of the examples given below is indicated in Fig. (1) by a small circle with the number of the corresponding example.

\section{Example 1}

The choice $A=-1 / 2$ and $B=C=0$ gives an $A$-stable method of the form

$\left(I-\frac{h}{2} f_{y}\right)\left[y_{1}(h)-y_{0}\right]=h f$

with $\sigma=-1 / 2$ in the principal term of the local error and the stability function

$R(z)=\frac{1+\frac{1}{2} z}{1-\frac{1}{2} z}$

Actually, this is a Rosenbrock type method, though it is not mentioned in [1].

\section{Example 2}

Now let us set $A=-1, B=-C=1 / 2$. In this case, we get an $L$-stable method 


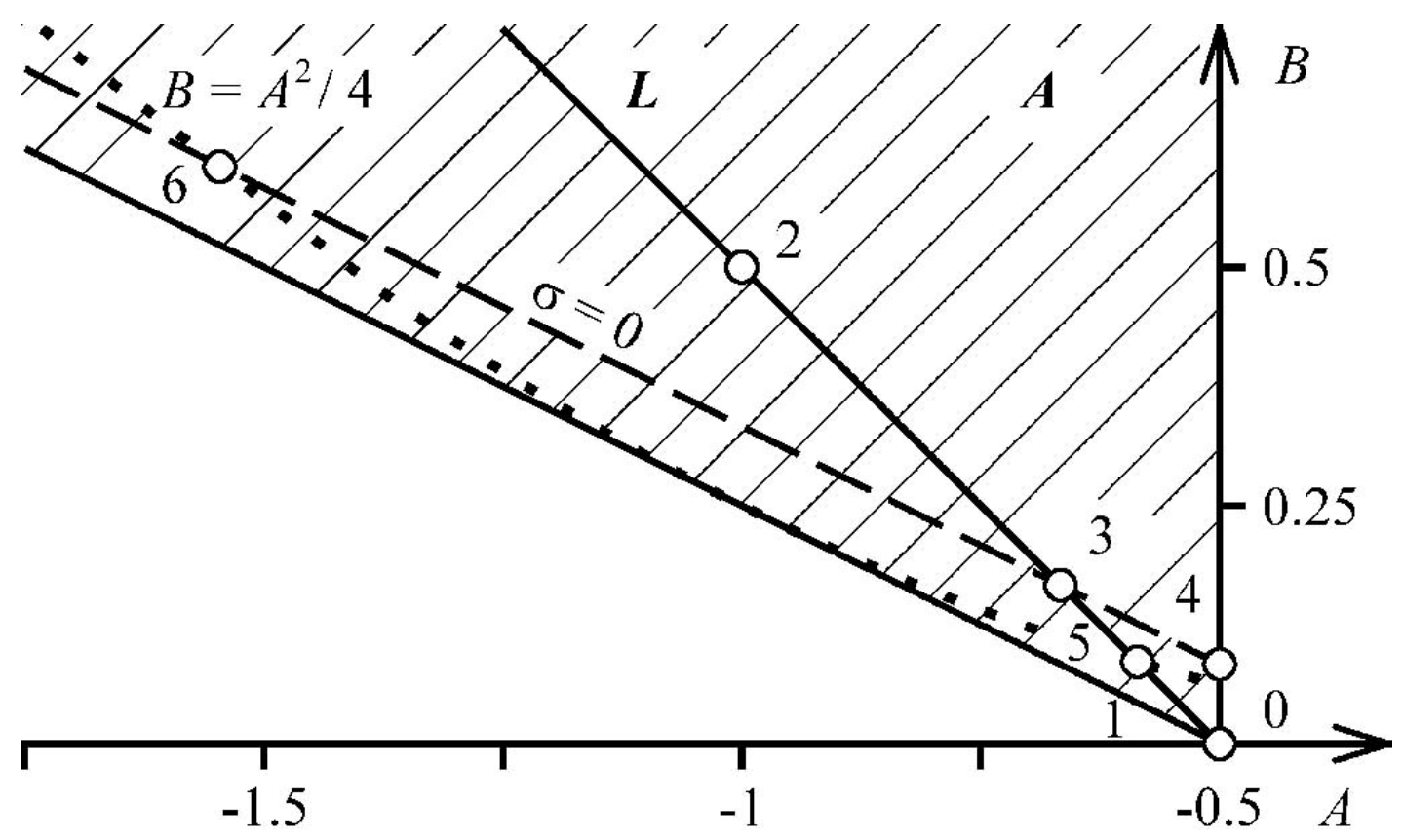

Fig. (1). $A$-stable and $L$-stable one-stage second order $A B C$-schemes.

$\left(I-h f_{y}+\frac{h^{2}}{2} f_{y}^{2}\right)\left[y_{1}(h)-y_{0}\right]=h f-\frac{h^{2}}{2} f_{y} f$

Eq. (3) gives $\sigma=1$ for this method, and the stability function of it is given by

$$
R(z)=\frac{1}{1-z+\frac{1}{2} z^{2}}
$$
[2].

The method was derived in other way and discussed in

\section{Example 3}

The choice $A=-2 / 3, B=-C=1 / 6$ gives the $L$-stable method

$$
\left(I-\frac{2 h}{3} f_{y}+\frac{h^{2}}{6} f_{y}^{2}\right)\left[y_{1}(h)-y_{0}\right]=h f-\frac{h^{2}}{6} f_{y} f
$$

with $\sigma=0$ in the principal term of local error. Therefore, this method is a member of the family defined by Eq. (4). Its stability function has the form

$$
R(z)=\frac{1+\frac{1}{3} z}{1-\frac{2}{3} z+\frac{1}{6} z^{2}}
$$

It was also mentioned in [3].

\section{Example 4}

The choice $A=-1 / 2, B=1 / 12$, and $C=0$ gives an $A$ stable method described in Corollary 2 (see above). The corresponding value of $\sigma$ from Eq. (3) is equal to zero. This method is also a member of the family described by Eq. (4). It gives fourth order approximation for the solutions of linear autonomous systems of differential equations.

\section{Example 5}

With the choice $B=A^{2} / 4$, we get 'cheap' $A B C$ schemes that minimize the costs of solving the system of linear algebraic equations (2) (see Remark 1 above). Then Eq. (2) takes the following form for the second order methods:

$$
\left(I+\frac{1}{2} A h f_{y}\right)^{2}\left[y_{1}(h)-y_{0}\right]=h f+\left(A+\frac{1}{2}\right) h^{2} f_{y} f
$$

Setting $A=-2+\sqrt{2} \approx-0.586$ gives an $L$-stable method. The stability function of this method is

$$
R(z)=\frac{1+(\sqrt{2}-1) z}{\left[1-\left(1-\frac{\sqrt{2}}{2}\right) z\right]^{2}}
$$

Eq. (3) yields the value of $\sigma$ equal to $4-3 \sqrt{2} \approx-0.243$.

\section{Example 6}

Another example of a 'cheap' $A B C$-scheme gives the choice $A=-1-3^{-1 / 2} \approx-1.577$

This time the value of $\sigma$ is equal to zero. This means that the method is also a member of the family described by Eq. (4), and it gives third order approximation for the solutions of linear autonomous systems of differential equations.

\section{MULTISTAGE $A B C$-SCHEMES}

\section{Definition 2}

A multistage $A B C$-scheme for numerical integration of a Cauchy problem for an autonomous system of $n$ ordinary differential equations (1) is defined as follows: 
$\left(I+A_{i} h f_{y}+B_{i} h^{2} f_{y}^{2}\right)\left[u_{i}(h)-y_{0}\right]$

$=\left(\alpha_{i} I+C_{i} h f_{y}\right) h f\left(u_{i-1}(h)\right)(i=1, \ldots . . s)$

$y_{1}(h)=\sum_{i=1}^{s} \beta_{i} u_{i}(h)$

$\sum_{i=1}^{s} \beta_{i}=1$

Here, $A_{i}, B_{i}, C_{i}, \alpha_{i}$, and $\beta_{i}$ are the coefficients that determine a particular method, $s$ is the number of stages $(s \geq 1), y_{1}(h)$ is the desired numerical solution after one step of integration with the step size $h\left(y_{1}(h)\right.$ is the weighted sum of partial solutions $u_{i}(h)$ obtained on the $i$ th stage, $\left.u_{0}(h) \equiv y_{0}\right) ; y(x)$ and $f(y)$ are $n$-dimensional vector functions, $f_{y}$ is the Jacobian matrix, and $I$ is the identity matrix. We consider the first step of integration as a representative one for the subsequent steps and write $f, f_{y}, \ldots$ without arguments for $f\left(y_{0}\right), f_{y}\left(y_{0}\right), \ldots$.

Note that the number of coefficients that define a particular multistage $A B C$-scheme in the case $s \geq 2$ is substantially more then for one-stage $A B C$-schemes. This fact enables one to construct methods of order higher then 2 , but it is also the cause of difficulties that encounter in the analysis of order conditions and stability functions.

\section{Theorem 6}

Stability functions of multistage $A B C$-schemes can be written in the following form:

$R(z)=\sum_{i=1}^{s} \beta_{i} R_{i}(z)$

The stability functions $R_{i}(z)$ of sequential stages are evaluated recursively:

$$
R_{i}(z)=1+\frac{P_{i}(z)}{Q_{i}(z)} R_{i-1}(z) \quad(i=1, \ldots, s)
$$

where

$$
R_{0}(z)=1, \quad P_{i}(z)=\alpha_{i} z+C_{i} z^{2}, \quad Q_{i}(z)=1+A_{i} z+B_{i} z^{2}
$$

The proof of this theorem is straightforward. One has to apply the formulas from Definition 2 to Dahlquist test equation $y^{\prime}=\lambda y$, where $\lambda$ is a complex number (see e.g. [5]), and then put $h \lambda=z$.

\section{EXAMPLES OF TWO-STAGE $A B C$-SCHEMES}

The gain of using 'cheap' $A B C$-schemes (see Remark 1 above) becomes still more in the case of multistage $A B C$ schemes. If we put $A_{i}=A, B_{i}=B$ for all stages and assume $B=A^{2} / 4$, then only a single $L U$-decomposition will be needed on each step of integration, since the matrix in the left-hand side of Eq. (5) is the same for all stages. For this reason, we confine ourselves with two examples of 'cheap' two-stage $A B C$-schemes.

\section{Example 1}

The choice $\alpha_{1}=\alpha_{2}=1, \beta_{1}=2 / 3, \beta_{2}=1 / 3, A_{1}=A_{2}=A$, $B_{1}=B_{2}=A^{2} / 4$ gives a family of two-stage third order methods depending on a single parameter $A$. In this case,

$C_{1}=-\frac{3}{4} A^{2}+\frac{1}{2} A \quad C_{2}=\frac{3}{2} A^{2}+2 A+\frac{1}{2}$

The stability function at $z \rightarrow \infty$ takes the form

$R(\infty)=-5+\frac{4}{A^{2}}+\frac{4}{3 A^{3}}$

These methods are $A$-stable at the values of $A$ between approximately -0.75 and -0.4 . The value $A \approx-0.59$ corresponds to an $L$-stable method.

\section{Example 2}

The choice $\alpha_{1}=1 / \sqrt{3}, \quad \alpha_{2}=1, \quad \beta_{1}=0, \quad \beta_{2}=1$, $A_{1}=A_{2}=A, B_{1}=B_{2}=A^{2} / 4$ gives again a family of twostage third order methods depending on a single parameter $A$, but in this case

$C_{1}=\frac{1}{4} A^{2}+\frac{1}{2} A+\frac{1}{2}-\frac{\sqrt{3}}{6} \quad C_{2}=A+\frac{1}{2}-\frac{\sqrt{3}}{3}$

and the stability function at $z \rightarrow \infty$ now takes the form

$$
R(\infty)=1+8\left[\frac{1}{A}+\left(\frac{3}{2}-\frac{\sqrt{3}}{3}\right) \frac{1}{A^{2}}+\left(\frac{1}{2}-\frac{\sqrt{3}}{3}\right) \frac{1}{A^{3}}+\left(\frac{5}{6}-\frac{1}{2} \sqrt{3}\right) \frac{1}{A^{4}}\right]
$$

Further results for these methods will be presented elsewhere.

\section{NUMERICAL EXPERIMENT}

For our numerical experiment, we have chosen a particular case of the singularly perturbed test problem suggested by Kaps [6], namely, the initial value problem

$$
\begin{aligned}
& y_{1}^{\prime}(x)=-\left(2+\varepsilon^{-1}\right) y_{1}(x)+\varepsilon^{-1} y_{2}^{2}(x), \\
& y_{2}^{\prime}(x)=y_{1}(x)-y_{2}(x)-y_{2}^{2}(x), \\
& y_{1}(0)=y_{2}(0)=1, \quad 0 \leq x \leq 1 .
\end{aligned}
$$

The exact solution of this problem $y_{1}(x)=e^{-2 x}, y_{2}(x)=e^{-x}$ does not depend on $\varepsilon$. However, the problem becomes very stiff, as $\varepsilon \rightarrow 0$.

We compare the results of numerical integration performed with the use of four methods:

- method 1 is the one-stage $A B C$-scheme from Example 3 of Section 3;

- method 2 is the implicit midpoint rule (one-stage Gauss method $[4,5])$;

- method 3 is the two-stage 'cheap' $A B C$-scheme from Example 1 of Section 5 with $A=-0.59$;

- method 4 is the two-stage Gauss method [5]. 
Table 1. The Comparison of Results Obtained with the Use of $A B C$-Schemes and Gauss Methods

\begin{tabular}{|c|c|c|c|c|c|c|c|c|}
\hline \multirow[b]{2}{*}{$\varepsilon$} & \multicolumn{2}{|c|}{ Method 1} & \multicolumn{2}{|c|}{ Method 2} & \multicolumn{2}{|c|}{ Method 3} & \multicolumn{2}{|c|}{ Method 4} \\
\hline & $\left\|e_{80}\right\|_{2}$ & $p_{a}$ & $\left\|e_{80}\right\|_{2}$ & $p_{a}$ & $\left\|e_{80}\right\|_{2}$ & $p_{a}$ & $\left\|e_{80}\right\|_{2}$ & $p_{a}$ \\
\hline $10^{-1}$ & $6.5 \cdot 10^{-6}$ & 2.1 & $1.1 \cdot 10^{-5}$ & 2.0 & $2.2 \cdot 10^{-7}$ & 2.9 & $6.3 \cdot 10^{-10}$ & 4.0 \\
\hline $10^{-2}$ & $9.5 \cdot 10^{-6}$ & 2.3 & $1.1 \cdot 10^{-5}$ & 2.0 & $1.6 \cdot 10^{-6}$ & 2.7 & $4.8 \cdot 10^{-9}$ & 4.0 \\
\hline $10^{-3}$ & $1.7 \cdot 10^{-5}$ & 2.2 & $1.1 \cdot 10^{-5}$ & 2.0 & $5.9 \cdot 10^{-6}$ & 2.2 & $4.7 \cdot 10^{-8}$ & 4.2 \\
\hline $10^{-4}$ & $2.1 \cdot 10^{-5}$ & 2.0 & $7.6 \cdot 10^{-6}$ & 2.8 & $8.1 \cdot 10^{-6}$ & 2.0 & $6.1 \cdot 10^{-7}$ & 4.6 \\
\hline $10^{-5}$ & $2.1 \cdot 10^{-5}$ & 2.0 & $2.2 \cdot 10^{-5}$ & 2.4 & $8.3 \cdot 10^{-6}$ & 2.0 & $6.9 \cdot 10^{-6}$ & 2.5 \\
\hline $10^{-6}$ & $2.1 \cdot 10^{-5}$ & 2.0 & $3.0 \cdot 10^{-5}$ & 2.0 & $8.3 \cdot 10^{-6}$ & 2.0 & $1.1 \cdot 10^{-5}$ & 2.0 \\
\hline $10^{-7}$ & $2.1 \cdot 10^{-5}$ & 2.0 & $3.0 \cdot 10^{-5}$ & 2.0 & $8.3 \cdot 10^{-6}$ & 2.0 & $1.1 \cdot 10^{-5}$ & 2.0 \\
\hline $10^{-8}$ & $2.1 \cdot 10^{-5}$ & 2.0 & $3.0 \cdot 10^{-5}$ & 2.0 & $8.3 \cdot 10^{-6}$ & 2.0 & $1.1 \cdot 10^{-5}$ & 2.0 \\
\hline
\end{tabular}

Methods 1, 2, 3, and 4 have classical order 2, 2, 3, and 4, respectively. These methods were used for the numerical integration of the above problem with several diminishing values of $\varepsilon$ and with two constant values of step size, $h=1 / 40$ and $h=1 / 80$. Table 1 contains the following values:

$\left\|e_{80}\right\|_{2}$ is the Euclidean norm of the absolute value of error for $h=1 / 80$ at the endpoint of the integration interval;

$p_{a}=\log _{2}\left(\left\|e_{40}\right\|_{2} /\left\|e_{80}\right\|_{2}\right)$ is the actual order of accuracy estimated using the results of integration with $h=1 / 40$ and $h=1 / 80$. In the case of methods 1 and 3 the computation was performed using double precision. In the case of methods 2 and 4 the data (evaluated with comparable precision) are taken from Table 7.5.2 in [7].

Observe that, for small values of $\varepsilon, A B C$-schemes give better results than implicit Runge-Kutta methods. Note that the implicit Runge-Kutta methods employ Newton iterations, i.e. they are more expensive then the $A B C$ schemes. One can clearly see the phenomenon of lowering of the actual order of accuracy at small values of $\varepsilon$ for the methods 3 and 4 , which is in accordance with the theory of $B$-convergence $[5,7]$.

\section{ACKNOWLEGEMENTS}

The authors are grateful to Professor J.G. Verwer for his encouraging criticism and to Professor E. Hairer for drawing our attention to a possible way to eliminate matrix multiplications. We are also thankful to Dr M.V. Bulatov for a useful discussion of one-stage $A B C$-schemes and to Dr G.Yu. Kulikov for his valuable observation.

\section{REFERENCES}

[1] Rosenbrock HH. Some general implicit processes for the numerical solution of differential equations. Comput J 1962/63; 5: 329-30.

[2] Bulatov MV. Construction of a one-stage $L$-stable second-order method. Differential Equations 2003; 39: 593-5.

[3] Filippov SS. $A B C$-schemes for stiff systems of ordinary differential equations. Doklady Mathematics 2004; 70: 878-80.

[4] Hairer E, Nørsett SP, Wanner G. Solving ordinary differential equations, I: Nonstiff problems. Springer-Verlag: Berlin 1993.

[5] Hairer E, Wanner G. Solving ordinary differential equations, II: Stiff and differential-algebraic problems. Springer-Verlag: Berlin 1996.

[6] Kaps P. Rosenbrock-type methods. In: Dahlquist G, Jeltsch R. Eds., Numerical methods for solving stiff initial value problems. Inst. für Geometrie und praktische Math. der RWTH Aachen 1981; Bericht No. 9.

[7] Dekker K, Verwer JG. Stability of Runge-Kutta methods for stiff nonlinear differential equations. North-Holland: Amsterdam 1984. 